\title{
L'évolution de la typologie de « la recette » au cours d'un siècle en Grèce
}

Des notes personnelles aux blogs culinaires

Modifications in the Generic Form and in the Appearance of "Recipes" from the End of the 19th Century in Greece

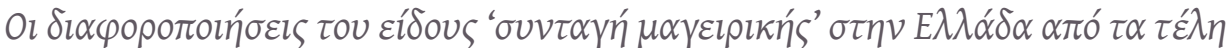

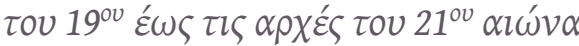

\section{Maria Papadopoulou}

\section{OpenEdition Journals}

Édition électronique

URL : https://journals.openedition.org/ceb/7186

DOI : $10.4000 /$ ceb. 7186

ISSN : 2261-4184

Éditeur

INALCO

Édition imprimée

ISBN : 978-2-85831-230-6

ISSN : 0290-7402

\section{Référence électronique}

Maria Papadopoulou, «L'évolution de la typologie de « la recette » au cours d'un siècle en Grèce », Cahiers balkaniques [En ligne], Hors-série | 2016, mis en ligne le 15 mars 2017, consulté le 06 juillet 2021. URL : http://journals.openedition.org/ceb/7186 ; DOI : https://doi.org/10.4000/ceb.7186

Ce document a été généré automatiquement le 6 juillet 2021.

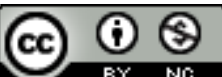

Cahiers balkaniques est mis à disposition selon les termes de la Licence Creative Commons Attribution - Pas d'Utilisation Commerciale 4.0 International. 


\section{L'évolution de la typologie de « la recette " au cours d'un siècle en Grèce}

Des notes personnelles aux blogs culinaires

Modifications in the Generic Form and in the Appearance of "Recipes" from the End of the 19th Century in Greece

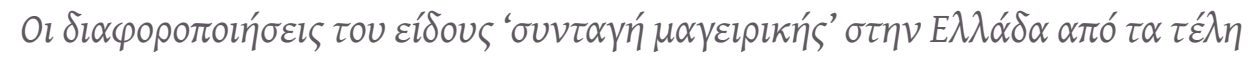

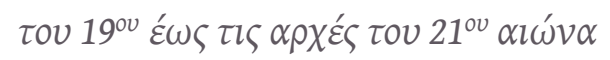

Maria Papadopoulou

\section{Introduction}

1 Ces dernières années, tant en Grèce que dans un certain nombre d'autres pays, l'on voit proliférer les éditions culinaires (ouvrages, magazines spécialisés) ainsi que les émissions télévisées quotidiennes sur la cuisine, voire des téléréalités, sans oublier les séminaires et les ateliers culinaires destinés aux non professionnels, les blogs culinaires sur l'internet, etc.

2 Le discours sur la nourriture se déplace donc du domaine privé au domaine public. D'occupation traditionnelle des femmes qu'elle était, la préparation des repas de la famille devient, dans le contexte du domaine public, une occupation de personnes de tous sexes et âges.

3 Cuisiner et traiter de nourriture et de repas semble aller au-delà d'une activité destinée à répondre à un besoin vital et à devenir l'élément d'un nouveau style de vie.

4 La diversification du genre « recette de cuisine », en Grèce, de la fin du XIX ${ }^{e}$ au début du $\mathrm{xxI}^{\mathrm{e}}$ siècle sera abordée dans le texte qui suit. On présentera la structure textuelle du genre et la fonction des divers modes de constitution du sens, afin de voir : a) si l'on 
peut observer des changements au cours d'un siècle, b) de quel type de changement il s'agit et c) si ces changements sont liés aux pratiques sociales concernant ce genre.

5 Le corpus est analysé à l'aide des grilles spécialisées. L'analyse s'étend aussi bien aux caractéristiques textuelles des recettes qu'aux traits multimodaux (typographie, couleur, images etc.), étant donné qu'au cours des années, les développements techniques concernant la typographie et les multimédias ont apporté des diversifications majeures à ce genre.

\section{Approches théoriques}

6 Comme on le sait grâce à la linguistique, la langue se concrétise par des types distincts d'énoncés, les genres textuels, qui permettent aux différents groupes sociaux de chaque époque d'atteindre leurs objectifs de communication (BAKHTIN, 1986). Si les genres n'existaient pas, si nous ne les maîtrisions pas, s'ils devaient être délimités au cours de la communication et que chaque énoncé devait être structuré pour la première fois, la communication linguistique serait quasiment impossible (BAKHTIN, 1986).

7 Dans l'effort de compréhension et de production d'un texte, la connaissance des typologies textuelles fournit aux lecteurs et producteurs un ensemble de stratégies qui les aident à résoudre des problèmes spécifiques (ADAM, 1999). Par conséquent, le genre agit tel un outil dans l'interprétation du texte et la formation des attentes concernant le développement du sujet en question (NYSTRAND, 1986). À l'instar de tous les systèmes sémiotiques dans chaque culture, les genres évoluent de manière à renforcer la stabilité, tout en étant flexibles afin de participer au changement social (MARTIN, CHRISTIE \& ROTHERY, 1994). Autrement dit, les genres recèlent un double caractère concomitant de stabilité et de différenciation. Les changements qui apparaissent dans les genres textuels découlent de leur adaptation à de nouvelles données sociales et à de nouveaux objectifs de communication (sWALES, 1990).

Les recettes de cuisine appartiennent au genre élargi de la "Procédure », tout comme les phases d'une expérience ou les instructions d'assemblage d'un objet : il y a toujours un but à atteindre et une description des étapes qui mènent à un résultat. Toutefois, comme on le verra par la suite, les différenciations du genre, en particulier à l'époque moderne, conduisent à l'incorporation de nouveaux micro-genres dans sa structure textuelle, afin de répondre à de nouveaux besoins sociaux de communication.

Les théoriciens des genres les ont essentiellement étudiés en tant que fruits de la production linguistique. Pourtant, ces dernières années, la pluralité des canaux et des modes de communication ainsi que la diversité culturelle linguistique des sociétés modernes ont induit des changements quant à la manière dont est conçue la communication entre citoyens (KALANTZIS \& COPE, 2000 ; KALANTZIS \& COPE, 2001; KRESS \& VAN LEEUWEN, 2006 ; KRESS, 2000 ; KRESS, 2010 ; FAIRCLOUGH, 2000). Par ailleurs, le déclin de la domination de la communication publique par la langue écrite en faveur des modes de représentation visuels - à l'instar de Kress (KRESS, 2000) - conduit à un réexamen de la notion de « texte écrit ». Compte tenu de sa multimodalité, il ne peut plus être atteint seulement par des savoirs et des savoir-faire de nature linguistique. Le message véhiculé par un texte ne renvoie pas exclusivement au contenu du scriptural (langue écrite) mais il émerge de la contribution réciproque des modes de représentation visuels utilisés (images, schémas, couleurs, typographie, etc.). Les textes reflètent ainsi 
l'authenticité de leur usage ; des images, la mise en page, la typographie, les couleurs etc. constituent à la fois des ressources sémantiques et des formes de représentations culturelles (KRESS 2010). Une telle définition du texte écrit fait apparaitre l'insuffisance $\mathrm{du}$ modèle classique «verbo-centrique» et suggère la nécessité d'étudier en profondeur la nature des textes multimodaux et des processus cognitifs qu'ils sollicitent.

10 La recherche sur la multimodalité a mis en lumière la diversité des modes de représentation qui y participent et qui interviennent de manière active à la constitution du sens.

\section{La méthodologie de la recherche}

11 Afin de répondre aux questions de la recherche, nous avons réuni des textes datant de la fin du XIX jusqu'au début du XXI ${ }^{e}$ siècle concernant une recette précise, à savoir, celle $\mathrm{du}$ « revani ». Les textes ont été choisis aléatoirement, en fonction de l'accessibilité des ressources concernées. Le corpus de la recherche se compose de dix textes: des manuscrits, des cahiers et des livres de cuisine, des revues générales ou spécialisées et des blogs culinaires.

Tableau 1. Le corpus

\begin{tabular}{|l|l|}
\hline le Brouillon d'Erato (Livre, auteur Lisa Micheli) (Fig. 1) & $\begin{array}{l}\text { Recettes de la fin du xixe siècle - } \\
\text { Début du xxe siècle. Reproduction } \\
\sim 1995\end{array}$ \\
\hline $\begin{array}{l}\text { la Nouvelle Cuisine de la maison (Livre, éditions M. Saliveros) } \\
\text { (Fig. 2) }\end{array}$ & Début 1950 \\
\hline Manuscrit 1 (archive personnelle) (Fig. 3) & Dans les années 1950 \\
\hline $\begin{array}{l}\text { Cuisine et pâtisserie illustrées (Livre, auteur Chrisa Paradisi) } \\
\text { (Fig. 4) }\end{array}$ & 1960 \\
\hline Manuscrit 2 (archive de Maria Poimenidou) (Fig. 5) & Dans les années 1960 \\
\hline Gourmet (Revue spécialisée, Journal Eleftherotypia) (Fig. 6) & 2006 \\
\hline $\begin{array}{l}\text { <https://www.sintagespareas.gr/sintages/rabani-aplo- } \\
\text { pentanostimo-afrato.html> }\end{array}$ & $2012(\mathrm{a})$ \\
\hline $\begin{array}{l}\text { <http://magyreuontas.blogspot.gr/2012/07/blog- } \\
\text { post_5800.html\#.Vbj06vmznPY> }\end{array}$ & $2012(\mathrm{~b})$ \\
\hline <http://www.funkycook.gr/siropiasto-revani-verias/> & 2013 \\
\hline $\begin{array}{l}\text { <http://www.seleo.gr/suntagh/178478-ravani-apo-proika- } \\
\text { tha-se-kano-magirissa\#.Vbj1GvmznPY> }\end{array}$ & 2015 \\
\hline
\end{tabular}


Figure 1 , Texte $\mathrm{n}^{\circ} 1$

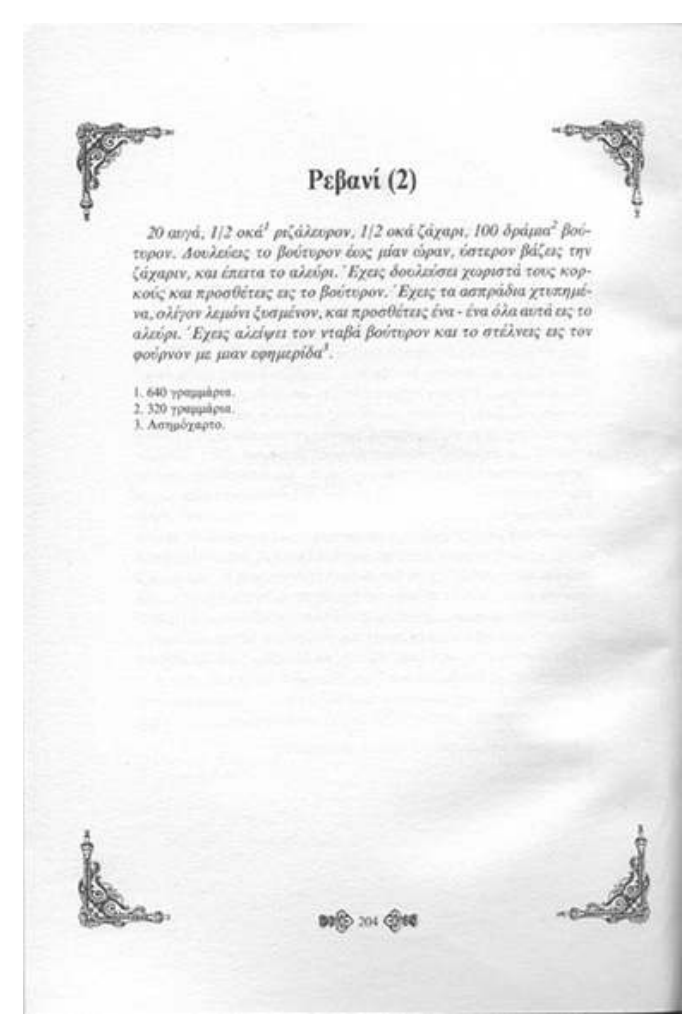

Le brouillon d'ERATO

Lisa Michel 
Figure 2, Texte $\mathrm{n}^{\circ} 2$

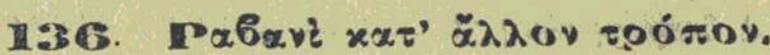

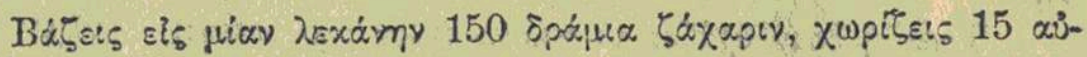

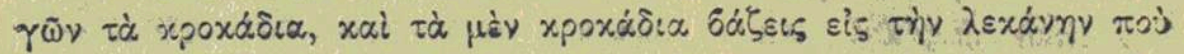

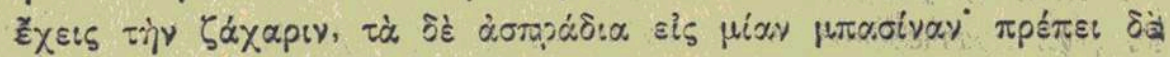

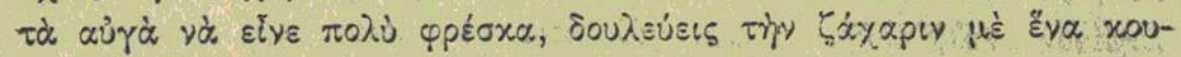

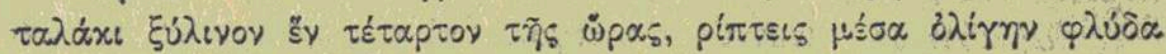

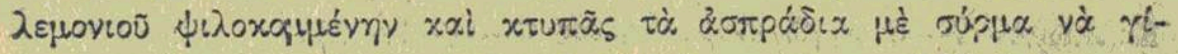

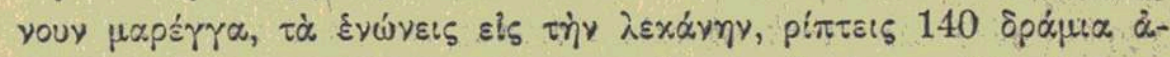

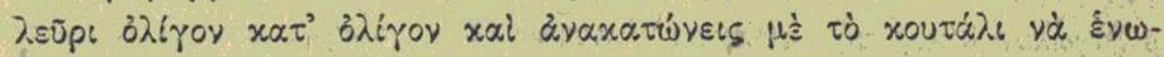

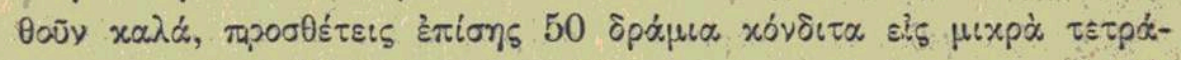

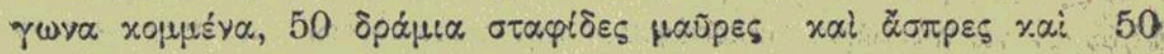

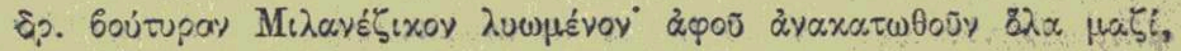

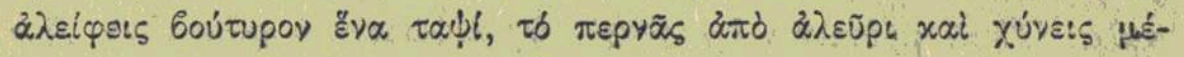

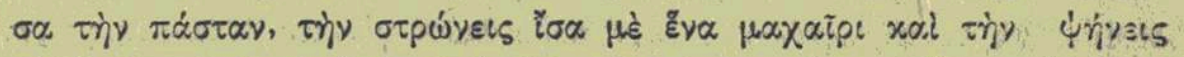

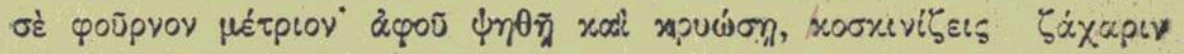

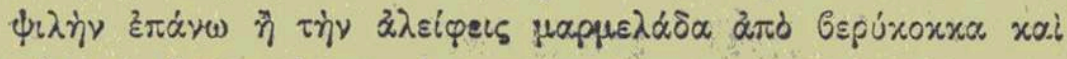

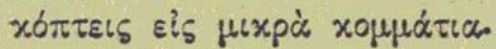

La nouvelle cuisine de la maison

Éditions M. Saliveros

Ensuite, on a enregistré tous les éléments de chacune des recettes et composé une grille d'analyse pour chacune des questions de la recherche, à savoir la structure du texte et la fonction des divers modes. Une fois les grilles construites, on a analysé chacun des textes. Nous avons ainsi réuni et codifié les données qui sont présentées dans les résultats et interprétées dans les conclusions. 
Figure 3 , Texte $\mathrm{n}^{\circ} 3$

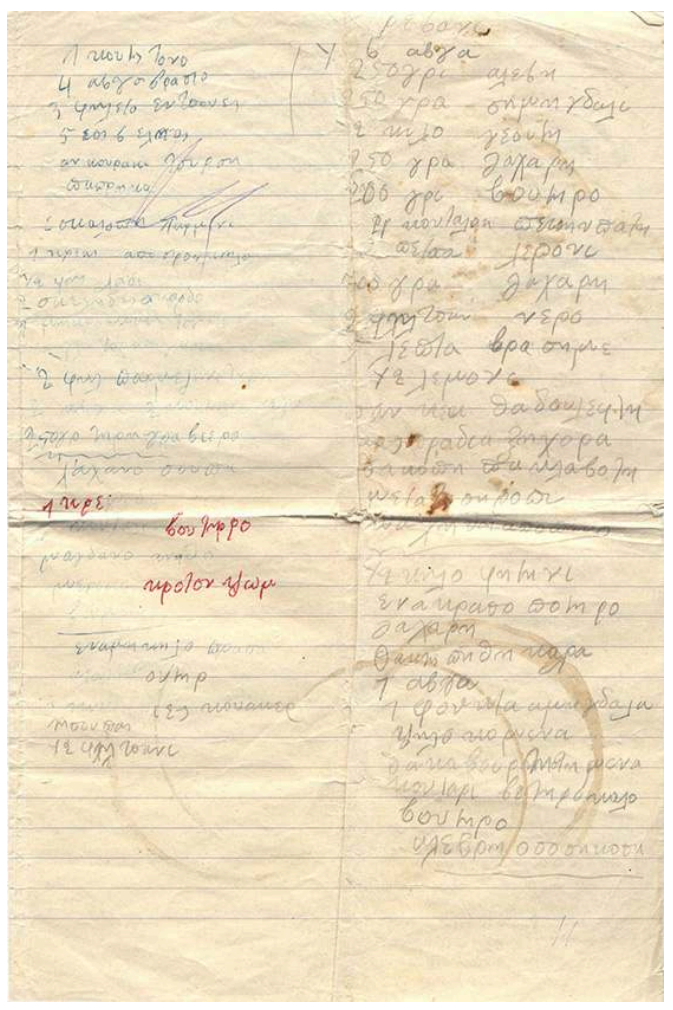

Archive personnelle

\section{Figure 4 , Texte $n^{\circ} 4$}

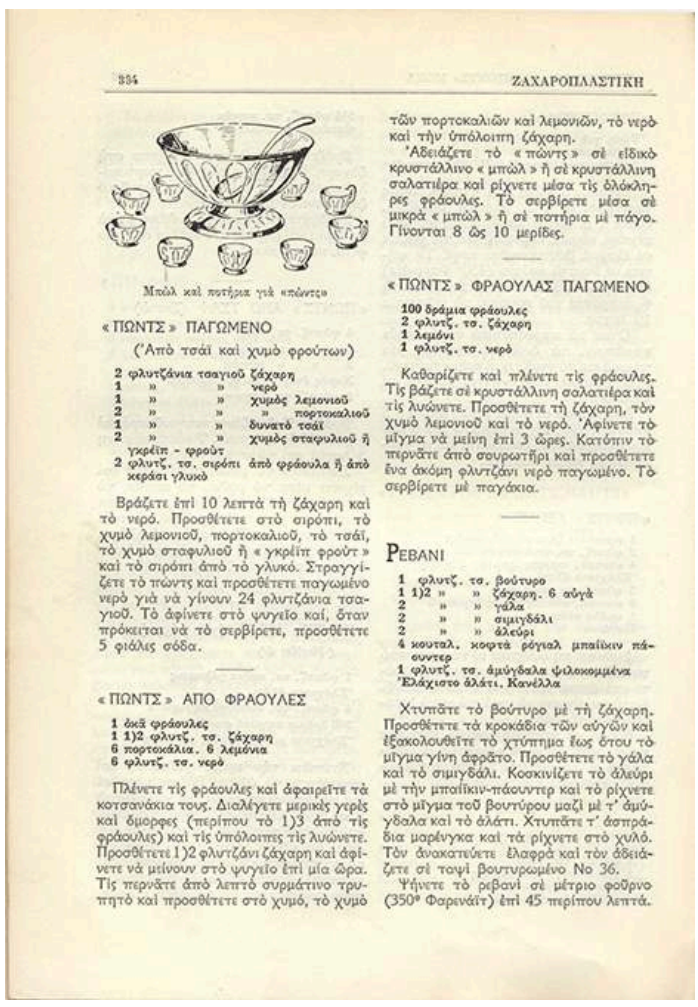

Cuisine et pâtisserie illustrées

Chrisa Paradisi 
Figure 5 , Texte $n^{\circ} 5$

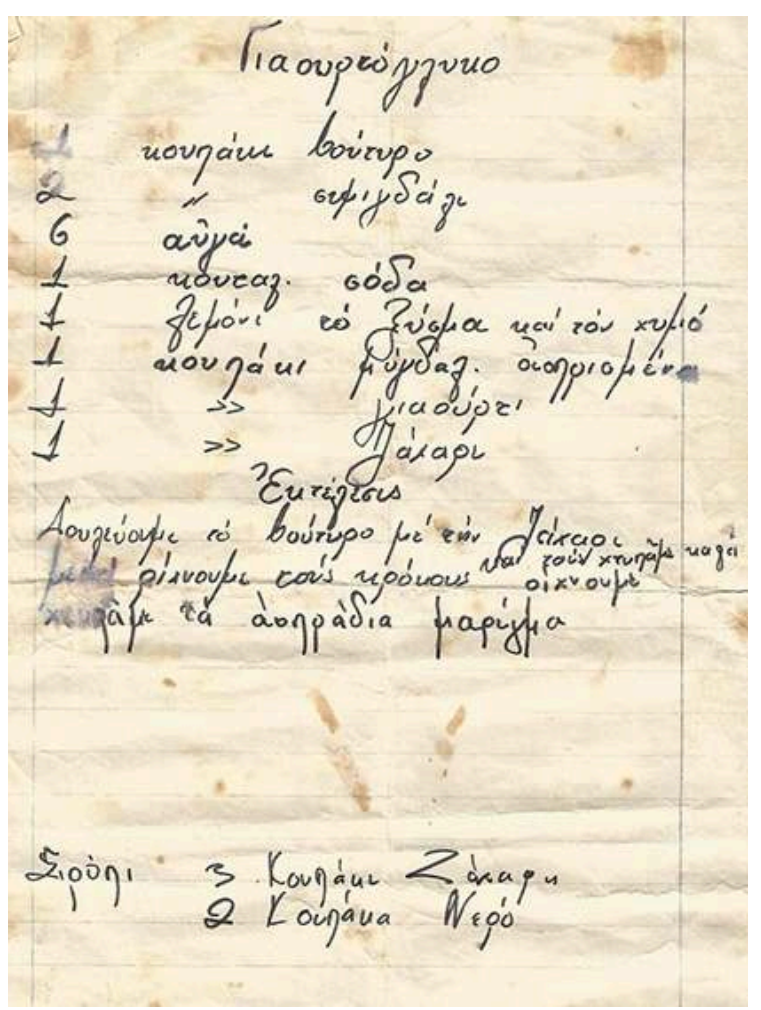

Archive Maria Poimenidou 
Figure 6, Texte $n^{\circ} 6$

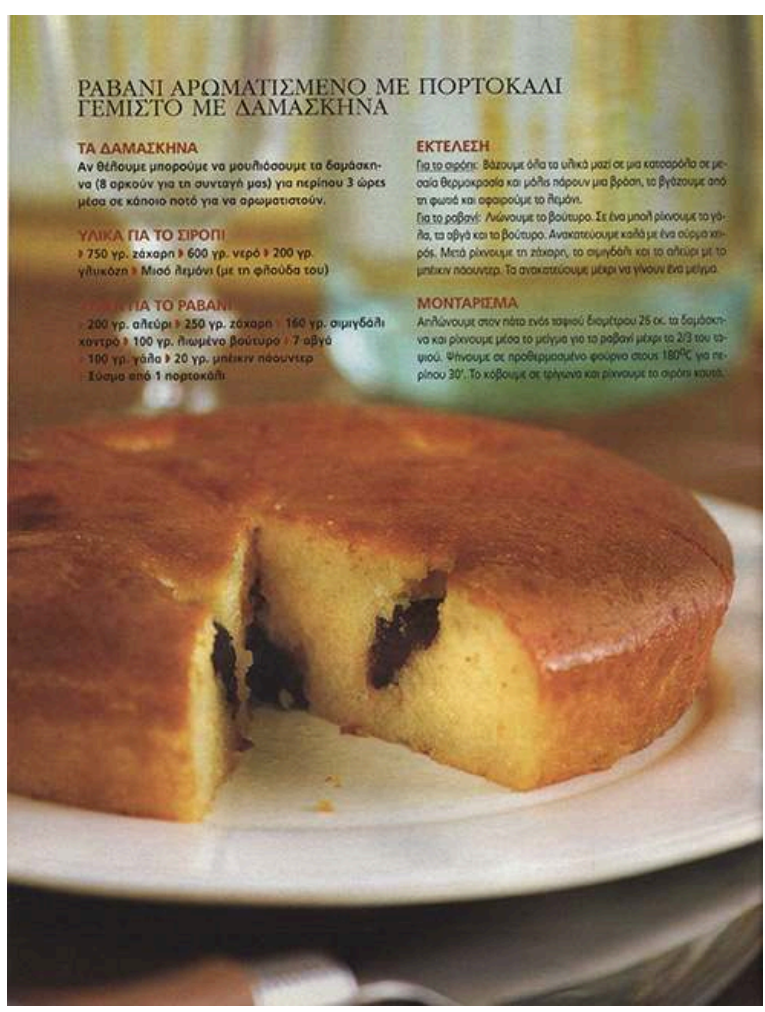

Gourmet

Eleftherotypia

\section{Grille d'analyse de la structure du texte}

13 La grille d'analyse concernant la structure du genre "recette de cuisine », a été élaborée sur la base des catégories individuelles identifiées dans l'ensemble des recettes qui composent l'échantillon. Dans la catégorie «détails», on a enregistré tous les genres supplémentaires qui apparaissent surtout dans les textes plus récents.

Tableau 2 : Structure générique

\begin{tabular}{|l|l|}
\hline Parties de la recette & Questions \\
\hline Créateur de la recette & Comment le créateur de la recette est-il présenté? \\
\hline $\begin{array}{l}\text { Catégorie } \\
\text { d'alimentation/Chapitres/Hyperliens }\end{array}$ & $\begin{array}{l}\text { Comment la catégorie à laquelle appartient la recette est- } \\
\text { elle présentée? Existe-t-il des hyperliens reliant des } \\
\text { différentes recettes? De quel type sont-ils? }\end{array}$ \\
\hline Titre de la recette & $\begin{array}{l}\text { Existe-t-il un titre? Où se trouve-t-il ? Comment se } \\
\text { différencie-t-il du reste du texte? }\end{array}$ \\
\hline Ingrédients & $\begin{array}{l}\text { Les ingrédients de la recette constituent-ils une partie } \\
\text { indépendante de la recette? Comment sont-ils disposés? } \\
\text { Comment se différencient-ils du reste du texte? }\end{array}$ \\
\hline
\end{tabular}




\begin{tabular}{|l|l|}
\hline Réalisation & $\begin{array}{l}\text { La réalisation de la recette constitue-t-elle une partie } \\
\text { autonome de la recette? Comment est-elle différenciée des } \\
\text { autres parties? Les instructions sont-elles détaillées ou } \\
\text { brèves? }\end{array}$ \\
\hline $\begin{array}{l}\text { Détails (temps de préparation, temps de } \\
\text { cuisson, coût, menu pour x personnes, } \\
\text { conseils, degré de difficulté, type } \\
\text { d'alimentation) }\end{array}$ & $\begin{array}{l}\text { Propose-t-on des «trucs » concernant la préparation de la } \\
\text { recette? Lesquels? Comment sont-ils présentés? }\end{array}$ \\
\hline $\begin{array}{l}\text { Commentaires par le créateur ou par } \\
\text { d'autres. }\end{array}$ & $\begin{array}{l}\text { Le créateur commente-t-il la recette? Est-elle commentée } \\
\text { par d'autres personnes? De quel type de commentaires } \\
\text { s'agit-il? Quel type d'interaction entre l'auteur et les } \\
\text { lecteurs révèlent-ils? }\end{array}$ \\
\hline
\end{tabular}

\section{Grille d'analyse de la fonction des divers modes}

14 Sur la base des dernières - du point de vue historique - versions du genre, c'est-à-dire des recettes présentées sur les blogs culinaires, il s'agit de textes multimodaux, dont l'attribution du sens résulte de la fonction de divers modes (langage, typographie, couleur, illustrations). Afin de connaître la contribution de chaque mode à la constitution du sens, on a élaboré la grille d'analyse suivante.

Tableau 3 : Distribution des modes

\begin{tabular}{|c|c|c|c|}
\hline \multicolumn{4}{|l|}{ Modes } \\
\hline \multirow[t]{2}{*}{ Langue } & Typographie & Couleur & Illustrations \\
\hline & $\begin{array}{l}\text { Différenciation (nulle, restreinte, } \\
\text { amplifiée). Préciser le type et la } \\
\text { fonction des choix } \\
\text { typographiques. }\end{array}$ & 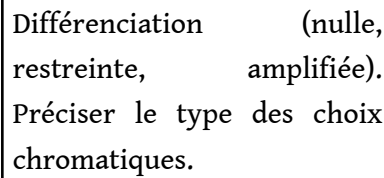 & $\begin{array}{l}\text { Aucune, } 1-2,3-4,5+ \\
\text { préciser le type } \\
\text { d'images présentées et } \\
\text { leur fonction. }\end{array}$ \\
\hline
\end{tabular}

\section{Résultats}

Selon les données reprises dans la grille d'analyse de la première question, les changements observés au cours d'un siècle portent principalement sur l'ajout de parties individuelles. Si la structure classique d'une recette de cuisine contient le titre, les ingrédients et le processus de préparation, dans les premiers textes de l'échantillon (jusqu'aux années 1950), on note la présence de seulement deux d'entre elles, c'est-àdire le nom de la recette et sa réalisation (les ingrédients sont intégrés dans la seconde partie). En outre, le nom de l'auteur de la recette est donné en couverture du livre. Une table des matières est également proposée au début ou à la fin de l'ouvrage. Il est intéressant de noter que les premiers textes sont caractérisés par l'information concentrée : ils sont brefs et concis. Il en va de même pour ce qui est des textes manuscrits. 
16 Au début du Xxi ${ }^{\mathrm{e}}$ siècle, les textes sont beaucoup plus longs, notamment dans les blogs culinaires. Le nom de la recette, les ingrédients, le processus de préparation ainsi que divers détails concernant le processus, c'est-à-dire, le coût de la recette, le temps nécessaire à la préparation, le temps de cuisson, le nombre de portions, le type d'alimentation, le degré de difficulté, etc. apparaissent dans des espaces distincts (Fig. 7).

Figure 7

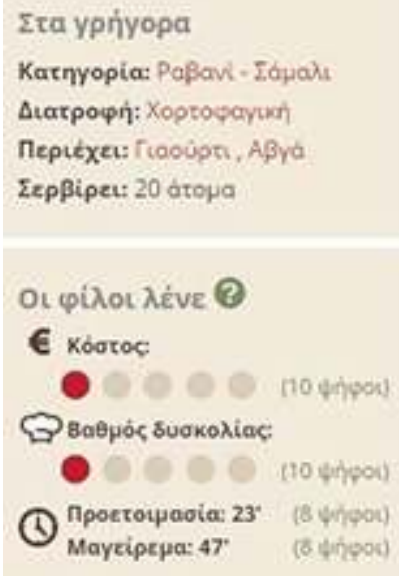

<https://www.sintagespareas.gr/sintages/rabani-aplo-pentanostimo-afrato.html>

Le média numérique permet aussi de créer des hyperliens qui servent à orienter le lecteur vers d'autres recettes proposées par l'auteur. Les hyperliens opèrent comme les tables de matières des livres de cuisine imprimés (Fig. 8)

Figure 8

You might also like:

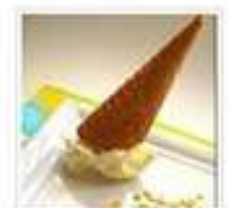

กavwió naoúpti

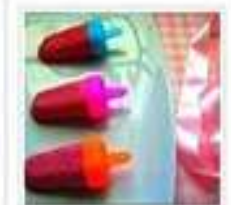

To mo uneivó mavwibll!

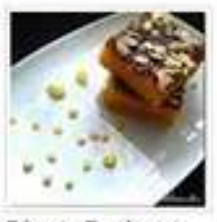

Eàuext nokinko!

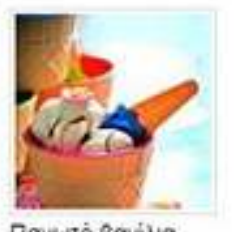

กoywió Bơvìia

Avoprịंenke amó Alexandra oा $12: 10 \mu \mu$

MDE[F⿻ 8+1 \&4 Mpoteiveri to oro Google

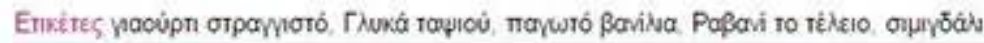

<http://magyreuontas.blogspot.gr/2012/07/blog-post_5800.html\#.Vbj06vmznPY>

Toutefois, l'élément le plus intéressant des recettes de cuisine contemporaines réside dans la présence de commentaires. Les commentaires de l'auteur sont particulièrement rares dans les textes de recettes plus anciens. Néanmoins, dans les blogs culinaires, ils 
occupent une place importante et, avec eux, apparaissent également les commentaires des lecteurs. Pour ce qui est de leur contenu, les commentaires des lecteurs concernent soit l'expression de leur approbation (qui, au-delà de l'expression verbale, s'exprime dorénavant à l'aide de votes, de petites fourchettes et de notifications sur Facebook), des questions posées à l'auteur afin d'obtenir des conseils individuels ou bien la présentation d'autres versions de la recette (Fig. 9). Nous aborderons de manière plus approfondie la fonction des commentaires dans les conclusions de la recherche.

Figure 9

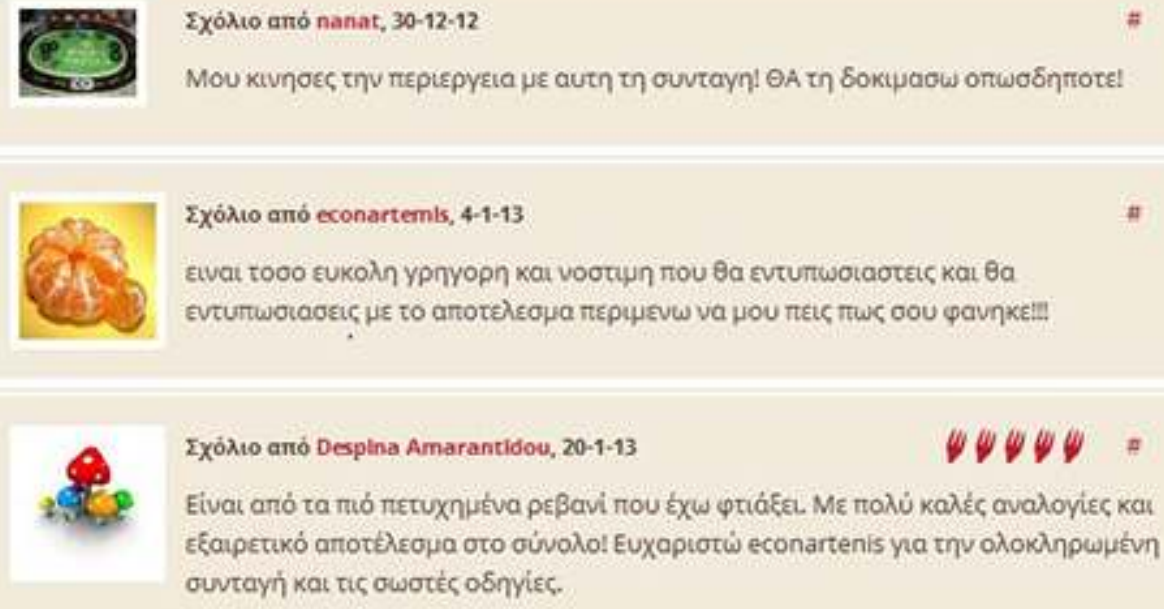

Exỏhı aró̉ Desplna Amarantidou, 20-1-13

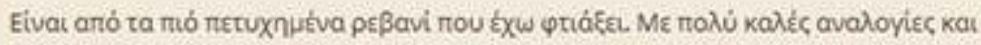

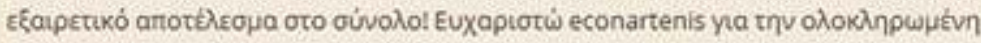
ouvtayń kat tic owotés osnyiec.

<http://www.sintagespareas.gr/sintages/rabani-aplo-pentanostimo-afrato.html>

L'analyse des données relevant de la deuxième question montre que, au fil du siècle, le genre « recette de cuisine » présente un changement radical des modes de constitution du sens. Dans les premiers textes, la langue constitue le mode fondamental et quasiunique des textes. L'on note également une faible attribution de sens par le biais de la typographie, en ce qui concerne le nom de la recette (caractères gras, de taille légèrement plus grande et centrés). Dans le dernier texte de l'échantillon, celui de 1960, la typographie est utilisée pour faire une distinction entre les diverses parties de la recette, c'est-à-dire les ingrédients et la réalisation de la recette (Fig.4). En outre, l'interlettrage plus large est également utilisé pour indiquer les divers niveaux de réalisation. Le même exemple que celui des livres est utilisé dans les deux textes manuscrits qui remontent au milieu du $\mathrm{xx}^{\mathrm{e}}$ siècle (Fig. 3, Fig. 5).

$\mathrm{Au}$ début du XxI ${ }^{\mathrm{e}}$ siècle, les textes se différencient fortement du point de vue des modes de constitution du sens. L'utilisation de photographies, notamment de photographies réalistes et, habituellement, en gros plan, « attirent » l'attention du lecteur et éveillent son intérêt pour réaliser la recette. La partie principalement affectée est celle qui concerne la réalisation de la recette (Fig. 10, page suivante).

Le discours écrit est significativement réduit. Néanmoins, il prévaut toujours dans la partie concernant les ingrédients de la recette qui se différencie du reste du texte à l'aide d'aménagements typographiques (utilisation de caractères gras, taille des lettres, disposition verticale) et, parfois, à l'aide de couleurs différentes. Les couleurs et les symboles sont également utilisés pour marquer plusieurs détails du processus (par 
exemple, temps de préparation et de cuisson, coût, portions, etc.) afin de les différencier du reste de la recette (Fig. 7).

Il est intéressant de noter que, bien que l'on observe une nette augmentation de l'information visuelle accompagnée d'une réduction correspondante de l'information linguistique, la langue revient dans les blogs culinaires pour devenir un élément qui caractérise surtout les recettes de cuisine contemporaines, c'est à dire les commentaires de la recette par l'auteur ou par les lecteurs.

Figure 10

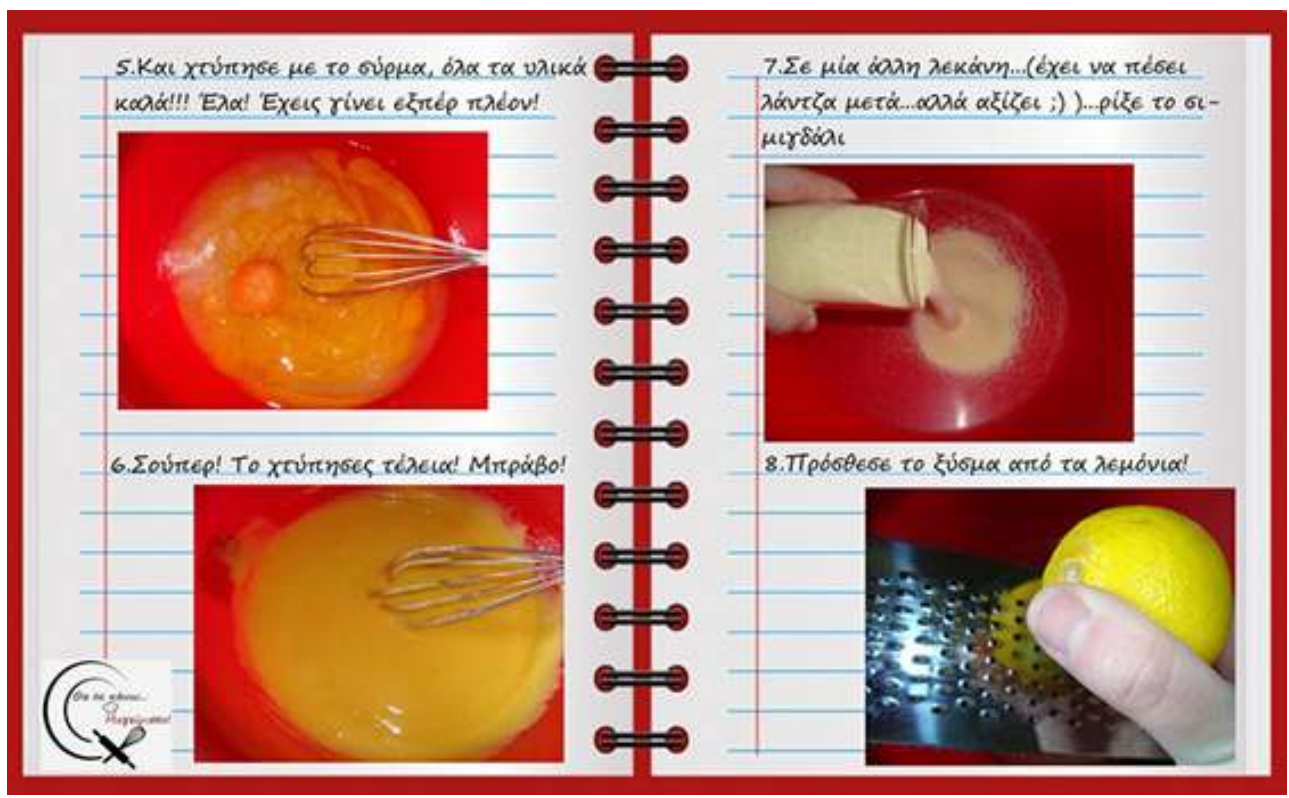

<http://www.seleo.gr/suntagh/178478-ravani-apo-proika-tha-se-kano-magirissa\#.Vbj1GvmznPY>

\section{Conclusions}

L'analyse des résultats de la recherche montre bien que, au fil d'un siècle, l'on observe des changements significatifs du genre de la recette, en Grèce. Ces changements concernent tant la structure textuelle du genre que la fonction des divers modes de constitution du sens.

En conclusion de cet article, j'entreprendrai une brève interprétation des résultats. La taille limitée des recettes dans les ouvrages du début du siècle ainsi que dans les textes manuscrits peut s'expliquer sur la base de leur public cible, à savoir les cuisinières expérimentées qui pouvaient réaliser une recette sans avoir besoin de nombreuses instructions et de détails pour réaliser un plat. Ce qui était surtout nécessaire, c'était de connaître le type et la quantité d'ingrédients. En outre, les recettes trop longues auraient entraîné une augmentation du volume du livre et, partant, de son prix. Au contraire, les recettes présentées sur l'internet ne sont pas affectées par cette restriction. Les recettes que l'on trouve sur les blogs culinaires sont très longues, surtout en ce qui concerne la partie contenant les instructions. Celles-ci sont proposées non seulement à l'aide de texte mais aussi de photographies. Ceci est probablement dû au fait que le public cible des auteurs des recettes ne se limite pas uniquement aux femmes au foyer expérimentées en la matière. Au contraire, l'internet est un outil de 
recherche pour un public plus large caractérisé par une relation plus "lâche " à la cuisine. $\mathrm{Si}$, au début du siècle, la cuisine ou la pâtisserie relevaient de la compétence exclusive de la femme au foyer qui ne travaillait pas en dehors de chez elle, dorénavant, un public de plus en plus large prépare occasionnellement des repas. Ce public n'est pas un familier de la cuisine, n'étant pas passé par un apprentissage dans la cuisine familiale. Il n'en reste pas moins qu'il revendique la possibilité de cuisiner, dans son temps libre, pour satisfaire ses besoins. Par conséquent, l'absence d'apprentissage rend nécessaire la présentation exhaustive et illustrée de toutes les étapes de réalisation d'une recette.

L'autorité de l'auteur, telle qu'elle est historiquement affirmée en couverture du livre de cuisine, est remplacée depuis quelques années par les créateurs des blogs qui publient leurs recettes pour leurs lecteurs. Il se crée ainsi un nouveau groupe dynamique "d'auteurs de recettes " nombreux qui, bien que n'étant pas «chefs » (à tout le moins, ils affirment ne pas l'être) souhaitent être reconnus dans le domaine, habituellement pour des raisons personnelles et/ou professionnelles (Fig. 11).

Figure 11

\section{Eva Movoxapn}

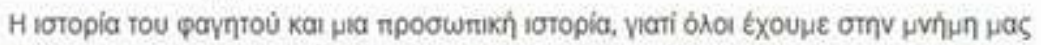

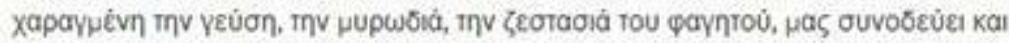

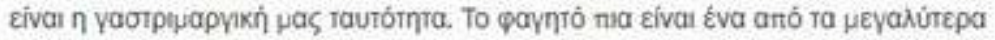

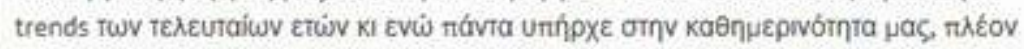

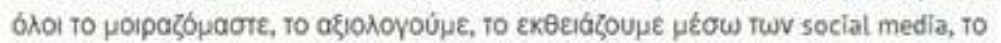

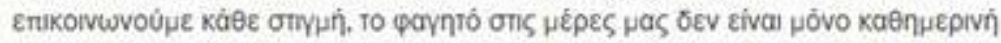

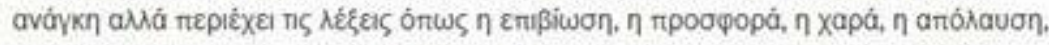

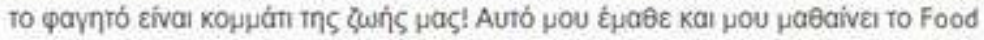

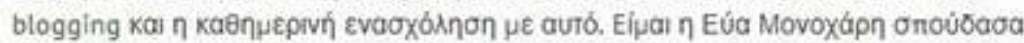

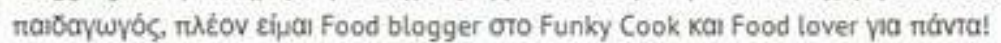

\section{f $g^{+}$or in $P$}

<http://www.funkycook.gr/siropiasto-revani-verias/>

Mais la réalisation de cet objectif exige la meilleure promotion de leurs recettes, de sorte qu'elles sont repérées et lues par une grande partie des lecteurs. À cela, outre le contenu des recettes, contribuent tant la variété des modes utilisés que leur acceptation par les lecteurs via les notifications sur Facebook, les commentaires positifs, etc.

27 La présence de commentaires répond, en même temps, à une fonction supplémentaire, à savoir celle de la création de réseaux sociaux dont l'objet est la cuisine. $\mathrm{Si}$, dans le temps historique du début du siècle, les recettes de cuisine faisaient l'objet de conversation entre les femmes au foyer, à l'époque contemporaine où ces conversations sont inexistantes ou limitées, les blogs offrent à des personnes de groupes sociaux, de genre et d'âge différents la possibilité de discuter entre eux en commentant une recette. Toutefois, l'observation attentive des commentaires des lecteurs montre que la discussion ne se limite pas à la recette et à sa réalisation, mais révèle également l'importance de la cuisine en tant que partie du quotidien et de la culture d'un peuple. C'est cette partie des recettes, c'est-à-dire les commentaires des lecteurs, qui permet de constituer un foyer numérique, que les membres numériques visitent pour créer des 
relations et restaurer une cohésion familiale souvent perdue dans les sociétés modernes.

Parmi les limitations de la recherche, il conviendra de noter qu'on n'a pas étudié de vidéos ni de recettes orales car la recherche était limitée aux seuls textes écrits (textes imprimés, manuscrits ou numériques). La taille relativement petite de l'échantillon (10 textes au total) constitue aussi l'une des limitations de la recherche.

\section{BIBLIOGRAPHIE}

ADAM Jean-Michel, 1992, les Textes : types et prototypes, Paris : Nathan.

BAKHTIN Mikhail, 1986, Speech Genres and other Late Essays, Texas: University of Texas Press.

FAIRCLOUG Norman, 2000, "Multiliteracies and language: Orders of Discourse and Intertextuality", in KALANTZIS Mary \& COPE Bill (dir.), Multiliteracies: The Design of Social Futures, London: Palmer Press.

KALANTZIS Mary \& COPE Bill (dir.), 2000, Multiliteracies: The Design of Social Futures, London: Palmer Press.

KALANTZIS Mary \& COPE Bill, 2001, “"Multiliteracies': A framework for Action”, in KALANTZIS Mary \& COPE Bill (dir.), Transformations in Language and Learning: Perspectives on Multiliteracies, Australia: Common Ground Publishing.

KRESS Gunther, 2000, "Design and Transformation: New Theories of Meaning", in KALANTZIS Mary \& cope Bill (eds), Multiliteracies: The Design of Social Futures, London: Palmer Press.

KRESS Gunther, 2010, Multimodality: A social semiotic approach to contemporary communication, London \& New York: Routledge.

KRESS Gunther, 2000, "Multimodality" in KALANTZIS Mary \& COPE Bill (dir.), Multiliteracies: The Design of Social Futures, London: Palmer Press.

KRESS Gunther \& van LEEUWEN Theo, 2006, Reading images: The grammar of visual design, London: Routledge.

MARTIN James Robert, CHRISTIE Francis \& ROTHERY Joan, 1994, “Social processes in Education: a Reply to Sawyer and Watson (and others)", in STIERER Barry \& MAYBIN Janet (dir.), Language, Literacy and Learning in Educational Practice, London: The Open University, pp. 232-247. NYSTRAND Martin, 1986, The structure of written communication: Studies in reciprocity between writers and readers, Orlando: Academic Press.

SWALES John, 1990, Genre Analysis, Cambridge: Cambridge University Press. 


\section{RÉSUMÉS}

La communication traite des multiples configurations des recettes de cuisine grecques de la fin du XIX ${ }^{\mathrm{e}}$ jusqu'au début du XXI ${ }^{\mathrm{e}}$ siècle. Elle présente la structure, la forme et les caractéristiques de ce genre de texte dans la diachronie, ainsi que les pratiques scripturaires et sociales dans lesquelles elle s'inscrit.

The paper discusses the ways the genre 'recipe' was transformed from the end of the 19th to the beginning of the 21st century in Greece. Modifications in the generic form and in the appearance of the text are presented along with the social practices into which recipes are involved.

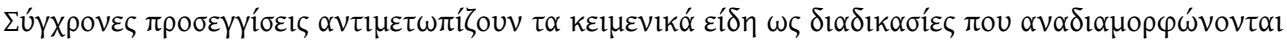

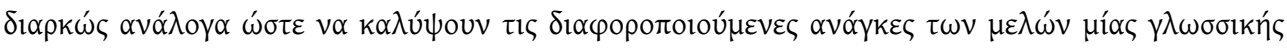

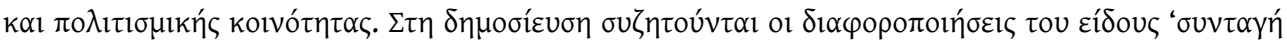

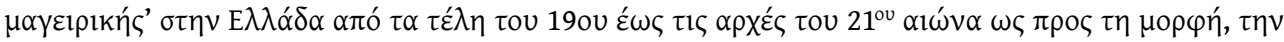

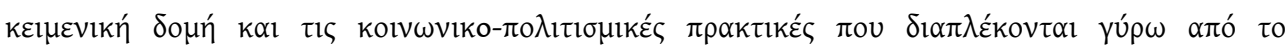

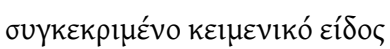

\section{INDEX}

Index géographique : Grèce

Mots-clés : recettes, recettes, cuisine, cuisine, pratiques socioculturelles, pratiques socioculturelles

Index chronologique : dix-neuvième siècle -- fin, vingtième siècle, vingt-et-unième siècle -début

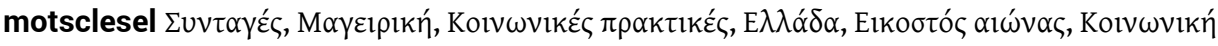

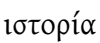

motsclestr Yemek Tarifleri, Gastronomi, sosyokültürel uygulamalar, Yunanistan, yirminci yüzyıl, Sosyal tarih

motsclesmk РЕЦЕПТИ, ГАСТРОНОМИЈА, СОЦИОКУЛТУРНИ ПРАКТИКИ, ГРЦИЈА, ДВАЕСЕТТИОТ ВЕК, СОЦИЈАЛНА ИСТОРИЈА

Keywords : Recipes, Cooking, Sociocultural practices, Greece, Twentieth Century, Social history

Thèmes : Histoire

\section{AUTEUR}

\section{MARIA PAPADOPOULOU}

Université de Thessalie 